\title{
Analysis of Health Care Products' Packaging Design under the Influence of Consumer Psychology
}

\author{
Xiaoli Fan \\ Huanghe Science and Technology College \\ Zhengzhou, China
}

\begin{abstract}
With the improvement of consumption level, people pay more attention to health with increasing demand for health care products, not only having higher requirements toward the quality of health care products, but also have new requirements for the packaging of health care products, namely the consumers' physical and psychological needs shall also be met in addition to satisfy the basic functions.
\end{abstract}

Keywords—con sumer psychology; packaging design of health care produces; influence; innovation

\section{INTRODUCTION}

There is a saying goes "sending healthy gifts", so the gist with concept of health has become the leading one for festive occasions. As a result health care product is the most appropriate choice. China is a state of ceremonies. An ancient saying goes "Friendship cannot stand always on one side", and it has been regarded as a classic Confucian etiquette. The packaging of health care products develops toward the highgrad trend due to the addition of culture of "etiquette". In order to pursue higher added value, there have been many "luxury package" and "excessive packaging", and such attractive packaging gives people a feeling of putting the cart before the horse, which is contrary to the original intention of packaging design.

With the change in consumer psychology, consumers have realized the importance of protecting the ecology, and are more eager to the green packaging, rather than the current packaging of "buying the casket without jewels". Therefore, this paper discusses the influence on packaging design of health care products by consumer psychology starting from the pers pective of consumer psychology.

\section{CONSUMER PSYCHOLOGY}

What is the consumer psychology? Consumer psychology refers to a series of consumer's mental activities in the process of purchasing, using and consuming the goods. Consumer psychology plays an increasingly important role in consumers' buying behavior. Therefore, the packaging of health care products need to be analyzed starting from consumers' purchase motivation. Only by grasping consumers' psychology, satisfying their physical and psychological needs, can the consumers generate feelings toward the packaging, thus become the loyal product consumers.

\section{PACKAGING DESIGN OF HEAlT H CARE PRODUCTS}

With the rise of supermarket, the promotion of health care products basically does not rely on salespeople, as packaging plays a role of "invisible salesman". Therefore, the packaging of health care products shall be able to attract the attention of consumers, arouse consumer buying desire, and leave a profound impression to consumers even if they don't buy, to play the role of publicizing the products.

With the increase of consumption level, consumers not only pay attention to product quality, but have raised higher requirements for product packaging. They pay more attention to the added value of packaging in addition to the basic protection function and convenient transportation, and they are increasingly willing to pay more money for "beautiful" packaging, which fully reflects the new trends of people's consumption in the new era. Thus it can be seen that good packaging plays a vital role for health care products.

\section{INFLUENCE ON PACKAGING DESIGN OF HEALT H CARE PRODUCTS BY CONSUMER PSYCHOLOGY}

\section{A. Packaging Design of Health Care Products Driven by the Consumer Psychology of Seeking Fame}

All kinds of consumer groups have a certain psychology of seeking fame, paying attention to the brand of goods and showing their status and prestige by virtue of well-known brands, thus to promote focusing more on brand imagebuilding of modern health care products' packaging design strategically.

The foothold and starting point of health care products' brand image-shaping is to conduct series packaging design for health care products. The so-called series packaging design refers to highlighting the integrity of visual form by virtue of the unified trademark image and text, or the same or similar color, pattern and arranged mode, and seek common points while reserving difference at the same time, to be of both diversity and unity.

With the continuous development of society, people pay more attention to health and have an increasingly demand for health care products. As a result, there are more and more kinds of health care products, with the increasingly fierce market competition. Series goods can increase consumers' attention, and the repeated appearance of a group of health care 
products' unified image can promote consumers to produce profound impression on the goods' names, trademarks, text, etc., which can not only attract consumers' attention in a very short period of time, and more importantly, it is able to establish the brand image in the minds of consumers successfully. Series packaging design has undergone a qualitative change, which is not just to use a unified form and unified color to regulate those products with different shapes, different uses but are intrinsically linked, but also the extension of enterprises' business philosophy, promoting wide spread of corporate culture and development of the brand, thus to meet consumers' demand for brand consumer.

\section{B. Packaging Design of Health Care Products Guided by the Consumer Psychology of Seeking Beauty}

With the rapid development of modern computer-aided design method, the expression means of health care products' packaging design are mostly computer special effects, which are known to be modern, too mechanical and lack of beauty. The pursuit of beauty is a basic feature of human psychology. In the modern fierce market competition, designers make efforts to pursue novel, special, strange, etc. packaging of health care products, but they often overlook that health care products packaging shall have the beauty that is corresponding to product characteristics.

Therefore, packaging design of health care products should meet the consumers' aesthetic taste, and improve the overall cultural taste of packaging design at the same time, so that consumers can obtain more and more aesthetic value in addition to enjoying the basic use value. The consumer psychology of seeking beauty needs the corresponding beautiful packaging design. Therefore, packaging design of health care products shall convey different goods' use value and aesthetic value by virtue of beautiful modeling, beautiful colors, beautiful material and beautiful styles to meet consumers' pursue of beauty.

\section{Packaging Design of Health Care Products Required by Emotional Consumer Psychology}

"It is emotion that first touches one", and specific to health care products packaging, the ability to attract consumers' attention is often closely linked with emotional activity. In fact, most of the emotional factors of the product itself are not strong or even extremely weak. As a result, the role of emotional factors shall be injected in packaging design in addition to paying attention to the intrinsic quality of goods . And the basic theory of emotional design is humanization.

The master of Bauhaus Nagy once said "the purpose of design is people, not products." We shall consider from the consumer's point of view and always take consumers' interest into consideration, to make consumers feel the designers' care and thought, thus to move the consumers, arouse their emotional response, and to cultivate consumers' emotion toward the commodities.

The main consumer group of health care products is the elderly. As China gradually steps into the "aging" society, the elderly has become a huge, social group cannot be ignored. Their consumption idea changes constantly, so more and more elderly people are willing to spend more money for their own health.

Different characteristics exist between the elderly and other consumer groups in terms of consuming behavior. Because the old people's physiological function degenerates gradually, their commodity consumption demand focuses on simplicity, convenience, and burden reduction in terms of physical and mental strength. So, the packaging of health care products for the elderly shall offer meticulous care for the elderly. The packaging of health care products for the elderly shall eliminate potential safety hazard first of all and avoid the too sharp articles to ensure safety use; second, the packaging shall not to be too complex for the convenience of the elderly to improve their self-confidence, strengthen their desire for activities and keep the independent living ability longer.

\section{Packaging Design of Health Care Products under the Call of Green Consumer Psychology}

In the early 20th century, with the continuous enrichment of social material wealth, serious environmental pollution and ecological crisis appear globally. Meanwhile, China is facing a series of ecological and environmental problems such energy shortage, desertification, species declining and decreasing arable land resources, which has seriously threaten people's lives, and made us fully aware that the deterioration of ecological environment shall be controlled, the previous bad spending habits shall be abandoned to promote green consumption.

Under the call of green consumer psychology, green packaging shall be adopted for health care products. Green packaging refers to the packaging that does not pollute the ecological environment and does not harm human health, which can be recycled and promote sustainable development. Its precondition is non-pollution and human health protection, with making full use of renewable resources, conserving natural resources and reducing energy consumption as the development orientation, thus forms the pattern of taking from nature and returning to nature. That is, the material it used is from the nature, and then forms into the green packaging products through non-polluting process. After using, it can be recycled for processing or returned to nature. As a result, green packaging contains two aspects of environmental protection and resource recycling.

Therefore, as for packaging of health care products, we shall minimize resource consumption and reduce pressure on environment to meet consumer demand based on meeting consumers' demand. Adopt the green packaging design, and reduce the damage on environment by packaging. Use the packaging material that can be reused or renewable as much as possible. Adopt the reasonable packaging structure, and fight against excessive packaging. 


\section{IMPROVEMENT MEASURES FOR PACKAGING DESIGN OF HEALT H CARE PRODUCT S UNDER THE INFLUENCE OF CONSUMER PSYCHOLOGY}

\section{A. Current Situation of Health Care Products Packaging Design}

Word is the main way to convey product information in the packaging of health care products. The name of health care products can be designed according to product attributes to make it closely integrate with the product and convey product information more vividly. The fonts of the same company's name are unified, to facilitate the identification by readers and the formation of serialization, as well as the establishment of brand image. However, the fonts on the package are changed a lot without unified words, which is difficult to be serialized and unable to satisfy consumers' psychology of branded consumption.

The successful packaging cannot be separated from personalized standard color. The successful application of packaging standard color can not only promote sales, brand image establishment, but also has the power of calling e motion. At present, the packaging design of entire gift box of the health care products is too complicated without theme tone, which cannot convey product attributes very well and is unable to give consumers the feeling of beauty. Thus it is difficult to arouse consumers' desire to buy, and is not conducive to sales of the products.

Graphics plays an important role in packaging design, which has become a major media to convey product information and stimulate the consumption. Therefore, the graphics in packaging should be typical, bright and be displayed on the box clearly. With the advent of information age, consumers receive a lot of information every day, and they need to find the products that are suitable for themselves from the vast information. Therefore, the packaging shall be able to attract their sight within a short time, which requires condensing the theme elements into a whole to display prominently. Currently, the packages on the market are short of theme graphics, which are too messy without a clear vision center, and it is difficult for consumers to obtain product information in a short time.

\section{B. Improvement Program for Packaging Design of Health Care Products}

Health care product is the enterprise and industry about health. Therefore, the reformation design shall be conducted for the packaging of health care products with the relaxed, elegant and healthy style as the theme, which is conducted mainly from the following aspects:

- As for the words, the words of brand in packaging shall use the unified font, and adjust the brand's position in the principal display surface at the same time to make it more prominent. The words in English are added on the packaging, which is beneficial to make the products access to international market.

- As for the color, design a tone that may give people the feeling of quietness symbolizing vigor and vitality, and it is easy for us to link it with health. Therefore, as a health-oriented enterprise, the products' natural characteristics shall be reflected on the whole packaging tone, to meet consumers' demand for green consumer psychology.

- As for the graphics, simplified treatment has been made for theme graphics. Hand-painted graphics is adopted, giving people a kind of intimacy, which is more easily recognized by consumers. At the same time, apply the products' raw materials to packaging design, enabling consumers to be able to judge products' attributes and related inform directly through vision. With the unified form and content, it is very easy to be favored by consumers.

- The simplified packaging design style is adopted. At the mention of health care products, we cannot help but link them with drugs. However, packaging design of health care products is as rational as that of the pure therapeutic drugs, as it can pursue richer effect in terms of vision according to the contents of the product itself. The packages of health care products on the market mostly focus on the feeling of similar to the drugs, which is too rational, and it is difficult to meet consumer pursuit of beautiful packaging. Therefore, I propose to change the packaging of health care products toward that of cosmetics, or to seek inspiration from the packaging design of cosmetics. Currently, the cosmetics packaging is mostly concise in terms of graphic design with prominent brand text, and the external packaging is mainly delicate color, which is full of the sense of beauty, meeting the consumers' psychology of seeking beauty, and has gained popularity. Currently, the packaging of health care products is too complicated. The improved packaging adopts the simplified style of cosmetics, advocate the use of clear and explicit form, pursue abundance in simplicity, and pursue elegance in pureness. At the same time, stress the design philos ophy of "less is more".

\section{CONCLUSION}

As the market economy continues to develop, consumers have become the leading factor in market economy. Only by accurately grasping consumers' needs and consumer psychology, can the orientation of packaging design be accurately grasped, can the products survive in the fierce market competition.

"In order to take, you shall give out first of all". In order to achieve the marketing purposes of health care products' packaging design, it is necessary to attract consumers' attention and to meet consumers' psychology. This needs to study the psychology of consumers, to consider from the consumers ' point of view and take the consumers' interest into consideration, arouse resonance among consumers, prompt consumers to develop feelings toward the products, meet their needs, stimulate consumer's potential consumption motivation, thus to move consumers from deep inside and make the m to be the loyal consumers of products. 


\section{REFERENCES}

[1] Chen Wenhua. Consumer Psychology and Marketing Strategies [M]. Beijing: China International Broadcast ing Publishing House, 2002.

[2] Fu Guoqun. Consumer Behavior Theory [M]. Beijing: Higher Education Press, 2007.

[3] Li Xiaoxia, Liu Jian. Consumer Psychology [M]. Beijing: Tsinghua University Press, 2007.

[4] Zhao Jianghong. Design Psychology [M]. Beijing: Beijing Institute of Technology Press, 2004.

[5] Guo Feng. Packaging Design [M]. Hangzhou: Zhejiang People's Fine Arts Publishing House, 1997.

[6] Wu Zuomin. Marketing [M]. Nanjing: Nanjing University Press, 2007.

[7] Guo Maolai. Artistic Appreciation of Packaging Design [M]. Beijing: People's Fine Arts Publishing House, 2001.

[8] Li Yanzu, Lu Ying. Graphic Design Art [M]. Beijing: China Renmin University Press, 2004. 